\title{
INDICADORES URBANOS E METROPOLITANOS
}

\section{1 ÍNDICE E SUBÍNDICES DE VULNERABILIDADE SOCIAL (2017-2018)}

A tabela 1 traz os dados relativos ao Índice de Vulnerabilidade Social (IVS) para as 27 Unidades da Federação (UFs) e para o país em 2017 e 2018, atualizando o acervo de indicadores publicados no Boletim Regional Urbano e Ambiental (Brua).

TABELA 1

Brasil: evolução do IVS para as UFs e o país (2017-2018)

\begin{tabular}{|c|c|c|}
\hline \multirow{2}{*}{ Região } & \multicolumn{2}{|c|}{ IVS } \\
\hline & 2017 & 2018 \\
\hline Brasil & 0,243 & 0,240 \\
\hline Distrito Federal & 0,258 & 0,251 \\
\hline Goiás & 0,247 & 0,234 \\
\hline Mato Grosso & 0,227 & 0,225 \\
\hline Mato Grosso do Sul & 0,194 & 0,192 \\
\hline Alagoas & 0,338 & 0,330 \\
\hline Bahia & 0,298 & 0,290 \\
\hline Ceará & 0,272 & 0,262 \\
\hline Maranhão & 0,349 & 0,349 \\
\hline Paraíba & 0,292 & 0,302 \\
\hline Pernambuco & 0,336 & 0,323 \\
\hline Piauí & 0,279 & 0,276 \\
\hline Rio Grande do Norte & 0,283 & 0,273 \\
\hline Sergipe & 0,298 & 0,306 \\
\hline Acre & 0,374 & 0,349 \\
\hline Amapá & 0,253 & 0,241 \\
\hline Amazonas & 0,327 & 0,330 \\
\hline Pará & 0,278 & 0,284 \\
\hline Rondônia & 0,191 & 0,199 \\
\hline Roraima & 0,232 & 0,256 \\
\hline Tocantins & 0,240 & 0,249 \\
\hline Espírito Santo & 0,227 & 0,216 \\
\hline Minas Gerais & 0,207 & 0,205 \\
\hline Rio de Janeiro & 0,284 & 0,278 \\
\hline São Paulo & 0,241 & 0,239 \\
\hline Paraná & 0,186 & 0,186 \\
\hline Rio Grande do Sul & 0,209 & 0,212 \\
\hline Santa Catarina & 0,134 & 0,129 \\
\hline
\end{tabular}

Fonte: Ipea, 2021.

1. DOI: http://dx.doi.org/10.38116/brua24art21 
É possível observar que, em 2018, os níveis mais elevados do IVS ainda se concentram na região Nordeste; entretanto, observou-se o aumento do índice na maioria das UFs da região Norte (Amazonas, Pará, Rondônia, Roraima e Tocantins), especialmente em Roraima, contrapondo-se à melhora observada no Acre, que, apesar disso, continua a ter o pior IVS do Brasil.

Os estados do Acre e do Maranhão apresentam os piores índices observados entre as UFs brasileiras (0,349), seguidos de Alagoas e Amazonas (0,330). O Maranhão manteve seu índice de 2017 e o Amazonas, que apresentou melhora em 2017, recuou ao patamar que havia sido observado em $2016 .^{2}$

Apesar de apresentar as UFs com os índices mais baixos, a regiăo Nordeste registrou melhora do IVS em seis estados. Apenas dois estados apresentaram piora no índice: Paraíba e Sergipe, os quais deixaram a faixa da baixa vulnerabilidade social para se situarem na faixa da média vulnerabilidade social.

$\mathrm{Na}$ tabela 2, que traz a análise para as dimensóes do IVS, é possível perceber que as regiôes Centro-Oeste, Sudeste e Sul detêm IVS Capital Humano (IVS-CH) e IVS Renda e Trabalho (IVS-RT) na faixa da baixa ou muito baixa vulnerabilidade social, ao passo que as regióes Norte e Nordeste concentram os maiores IVS-CH e IVS-RT.

TABELA 2

Brasil: evolução das dimensões do IVS para as UFs e para o país (2017-2018)

\begin{tabular}{|c|c|c|c|c|c|c|}
\hline \multirow{2}{*}{ Região } & \multicolumn{3}{|c|}{2017} & \multicolumn{3}{|c|}{2018} \\
\hline & IVS-IU & IVS-CH & IVS-RT & IVS-IU & IVS-CH & IVS-RT \\
\hline Brasil & 0,204 & 0,226 & 0,300 & 0,203 & 0,221 & 0,295 \\
\hline Distrito Federal & 0,405 & 0,171 & 0,198 & 0,403 & 0,152 & 0,198 \\
\hline Goiás & 0,270 & 0,218 & 0,253 & 0,269 & 0,205 & 0,228 \\
\hline Mato Grosso & 0,229 & 0,219 & 0,232 & 0,230 & 0,222 & 0,221 \\
\hline Mato Grosso do Sul & 0,134 & 0,209 & 0,239 & 0,136 & 0,219 & 0,220 \\
\hline Alagoas & 0,166 & 0,386 & 0,460 & 0,167 & 0,371 & 0,451 \\
\hline Bahia & 0,137 & 0,321 & 0,435 & 0,134 & 0,305 & 0,430 \\
\hline Ceará & 0,158 & 0,294 & 0,363 & 0,150 & 0,281 & 0,354 \\
\hline Maranhão & 0,272 & 0,336 & 0,440 & 0,256 & 0,335 & 0,456 \\
\hline Paraíba & 0,189 & 0,313 & 0,375 & 0,189 & 0,332 & 0,385 \\
\hline Pernambuco & 0,273 & 0,306 & 0,430 & 0,273 & 0,286 & 0,410 \\
\hline Piauí & 0,138 & 0,298 & 0,400 & 0,127 & 0,295 & 0,405 \\
\hline Rio Grande do Norte & 0,171 & 0,281 & 0,398 & 0,170 & 0,282 & 0,368 \\
\hline Sergipe & 0,142 & 0,321 & 0,431 & 0,144 & 0,315 & 0,458 \\
\hline Acre & 0,335 & 0,366 & 0,420 & 0,322 & 0,343 & 0,383 \\
\hline Amapá & 0,071 & 0,303 & 0,386 & 0,057 & 0,269 & 0,396 \\
\hline Amazonas & 0,318 & 0,283 & 0,379 & 0,320 & 0,294 & 0,374 \\
\hline Pará & 0,171 & 0,287 & 0,377 & 0,170 & 0,288 & 0,394 \\
\hline Rondônia & 0,058 & 0,260 & 0,254 & 0,057 & 0,257 & 0,283 \\
\hline Roraima & 0,197 & 0,231 & 0,269 & 0,197 & 0,259 & 0,311 \\
\hline Tocantins & 0,173 & 0,242 & 0,306 & 0,177 & 0,268 & 0,300 \\
\hline Espírito Santo & 0,183 & 0,217 & 0,280 & 0,185 & 0,197 & 0,264 \\
\hline Minas Gerais & 0,129 & 0,201 & 0,291 & 0,128 & 0,205 & 0,280 \\
\hline Rio de Janeiro & 0,407 & 0,173 & 0,271 & 0,406 & 0,165 & 0,263 \\
\hline São Paulo & 0,328 & 0,157 & 0,239 & 0,328 & 0,152 & 0,236 \\
\hline Paraná & 0,163 & 0,184 & 0,212 & 0,168 & 0,186 & 0,203 \\
\hline Rio Grande do Sul & 0,238 & 0,174 & 0,214 & 0,240 & 0,178 & 0,218 \\
\hline Santa Catarina & 0,098 & 0,149 & 0,156 & 0,098 & 0,142 & 0,146 \\
\hline
\end{tabular}

Fonte: Ipea, 2021.

2. Disponível em: <https://bit.ly/3fFNbaU>. 
Destacam-se os mais altos subíndices do país nas UFs de Alagoas, Maranhão e Sergipe, em 2018, e o aumento do IVS-CH, para o período 2017-2018, em Roraima e Tocantins, mantendo-os na faixa de baixa vulnerabilidade social associada ao capital humano.

Roraima também apresentou um aumento expressivo no IVS-RT, culminando, nesse caso, na mudança para a faixa de média vulnerabilidade social na dimensão de renda e trabalho.

Conforme se pode observar na tabela 2, o IVS Infraestrutura Urbana (IVS-IU) é a dimensão com maior desigualdade entre as UFs, em relação às suas Grandes Regiôes, apresentando o melhor desempenho geral.

O Amapá, que já apresentava o segundo IVS-IU mais baixo do país, evoluiu ainda mais e alcançou o IVS-IU de Rondônia. Apesar da melhora discreta do IVS-IU no Distrito Federal e no Rio de Janeiro, as duas UFs ainda possuem os maiores subíndices da categoria no país. O Amapá também apresentou a melhora mais expressiva do IVS-CH, seguido do Acre, que se destacou positivamente, reduzindo os subíndices de vulnerabilidade em cada uma das três dimensóes, apesar de ainda possuir altos IVS-UI e IVS-CH.

\section{DESAGREGAÇÕES DO IVS (2017-2018)}

As próximas tabelas trazem o IVS com os dados desagregados por sexo, cor e situação de domicílio selecionados: população feminina (tabela 3), brancos e negros (tabela 4) e para a população urbana (tabela 5).

$\mathrm{Na}$ tabela 3, que diz respeito à desagregação em relação à populaçáo feminina, observou-se uma pequena retração do índice em UFs que apresentam IVS mais elevados, a saber: Alagoas, Maranhão e Amazonas.

Em 2018, a melhora do IVS para as mulheres no Mato Grosso e na Bahia garantiu a evoluçáo de suas classificaçôes para as faixas de muito baixa e baixa vulnerabilidade social, respectivamente. Destaca-se, também, a evoluçáo do índice observado no Acre, ainda que sua classificação, em 2018, tenha se mantido na faixa de média vulnerabilidade social.

Ainda que a região Nordeste apresente os índices mais elevados também para o recorte da populaçáo feminina, a regiáo Norte foi a que mostrou o maior aumento do IVS no período. Vale dizer que a região reúne estados com IVS na faixa da média vulnerabilidade social (Acre e Amazonas), mas também traz o caso de Rondônia, onde o IVS está na faixa da muito baixa vulnerabilidade social e é um dos menores do país.

Ainda na regiáo Norte, fica o destaque negativo para Roraima, onde o aumento do IVS da população feminina fez com que a UF caísse uma faixa da vulnerabilidade social.

O IVS da população feminina mais baixo do país encontra-se em Santa Catarina $(0,127)$, e as UFs de Mato Grosso, Mato Grosso do Sul, Paraná e Rio Grande do Sul, além do estado de Rondônia, encontram-se na mesma faixa de muito baixa vulnerabilidade social. 
TABELA 3

Brasil: evolução do IVS das mulheres para as UFs e para o país (2017-2018)

\begin{tabular}{|c|c|c|}
\hline \multirow{2}{*}{ Região } & \multicolumn{2}{|c|}{ IVS } \\
\hline & 2017 & 2018 \\
\hline Brasil & 0,252 & 0,247 \\
\hline Distrito Federal & 0,269 & 0,258 \\
\hline Goiás & 0,273 & 0,263 \\
\hline Mato Grosso & 0,203 & 0,198 \\
\hline Mato Grosso do Sul & 0,196 & 0,194 \\
\hline Alagoas & 0,344 & 0,338 \\
\hline Bahia & 0,303 & 0,293 \\
\hline Ceará & 0,277 & 0,259 \\
\hline Maranhão & 0,334 & 0,331 \\
\hline Paraíba & 0,279 & 0,293 \\
\hline Pernambuco & 0,335 & 0,323 \\
\hline Piauí & 0,257 & 0,257 \\
\hline Rio Grande do Norte & 0,287 & 0,268 \\
\hline Sergipe & 0,300 & 0,311 \\
\hline Acre & 0,353 & 0,324 \\
\hline Amapá & 0,253 & 0,236 \\
\hline Amazonas & 0,331 & 0,328 \\
\hline Pará & 0,287 & 0,293 \\
\hline Rondônia & 0,178 & 0,189 \\
\hline Roraima & 0,198 & 0,226 \\
\hline Tocantins & 0,214 & 0,224 \\
\hline Espírito Santo & 0,260 & 0,245 \\
\hline Minas Gerais & 0,218 & 0,212 \\
\hline Rio de Janeiro & 0,295 & 0,291 \\
\hline São Paulo & 0,272 & 0,269 \\
\hline Paraná & 0,198 & 0,194 \\
\hline Rio Grande do Sul & 0,185 & 0,189 \\
\hline Santa Catarina & 0,133 & 0,127 \\
\hline
\end{tabular}

Fonte: Ipea, 2021.

A tabela 4, por seu turno, traz o IVS para a população autodeclarada branca ou preta nas UFs do país, permitindo apontar as oscilaçóes ocorridas entre 2017 e 2018, segundo os dados da Pesquisa Nacional por Amostra de Domicílios (PNAD), realizada pelo Instituto Brasileiro de Geografia e Estatística (IBGE). 
TABELA 4

Brasil: evolução do IVS para as UFs e para o país por cor (2017-2018)

\begin{tabular}{|c|c|c|c|c|}
\hline \multirow{2}{*}{ Região } & \multicolumn{2}{|c|}{ Brancos } & \multicolumn{2}{|c|}{ Negros } \\
\hline & 2017 & 2018 & 2017 & 2018 \\
\hline Brasil & 0,201 & 0,198 & 0,279 & 0,273 \\
\hline Distrito Federal & 0,226 & 0,230 & 0,278 & 0,265 \\
\hline Goiás & 0,195 & 0,189 & 0,269 & 0,251 \\
\hline Mato Grosso & 0,180 & 0,195 & 0,244 & 0,233 \\
\hline Mato Grosso do Sul & 0,137 & 0,142 & 0,222 & 0,214 \\
\hline Alagoas & 0,302 & 0,274 & 0,349 & 0,349 \\
\hline Bahia & 0,236 & 0,259 & 0,312 & 0,296 \\
\hline Ceará & 0,234 & 0,230 & 0,287 & 0,274 \\
\hline Maranhão & 0,313 & 0,299 & 0,358 & 0,361 \\
\hline Paraíba & 0,293 & 0,292 & 0,297 & 0,311 \\
\hline Pernambuco & 0,286 & 0,276 & 0,358 & 0,345 \\
\hline Piauí & 0,227 & 0,213 & 0,291 & 0,290 \\
\hline Rio Grande do Norte & 0,244 & 0,226 & 0,305 & 0,299 \\
\hline Sergipe & 0,248 & 0,269 & 0,310 & 0,315 \\
\hline Acre & 0,346 & 0,297 & 0,377 & 0,360 \\
\hline Amapá & 0,226 & 0,195 & 0,259 & 0,250 \\
\hline Amazonas & 0,245 & 0,229 & 0,344 & 0,350 \\
\hline Pará & 0,255 & 0,253 & 0,283 & 0,291 \\
\hline Rondônia & 0,186 & 0,181 & 0,195 & 0,208 \\
\hline Roraima & 0,250 & 0,275 & 0,228 & 0,251 \\
\hline Tocantins & 0,199 & 0,184 & 0,252 & 0,263 \\
\hline Espírito Santo & 0,185 & 0,180 & 0,252 & 0,236 \\
\hline Minas Gerais & 0,172 & 0,173 & 0,230 & 0,224 \\
\hline Rio de Janeiro & 0,244 & 0,242 & 0,310 & 0,303 \\
\hline São Paulo & 0,215 & 0,210 & 0,278 & 0,277 \\
\hline Paraná & 0,176 & 0,173 & 0,215 & 0,216 \\
\hline Rio Grande do Sul & 0,184 & 0,188 & 0,274 & 0,273 \\
\hline Santa Catarina & 0,118 & 0,116 & 0,196 & 0,171 \\
\hline
\end{tabular}

Fonte: Ipea, 2021.

Em nível nacional, a classificação da população branca em termos das faixas do IVS permitiu uma mudança de faixa, passando o índice para a faixa da muito baixa vulnerabilidade social. O IVS da populaçáo negra, embora tenha permanecido na mesma faixa de 2017 , registrou uma reduçấo mais intensa.

$\mathrm{Na}$ análise dos dados por UF, ainda que tenhamos observado uma mudança muito positiva nos índices para a população autodeclarada branca nos estados de Acre, Amapá, Alagoas e Maranhão, repercutindo na conquista de classificaçóes melhores no IVS, três grandes aumentos foram observados na Bahia, no Sergipe e em Roraima.

A melhora do índice mais expressiva para a população autodeclarada negra ocorreu em Santa Catarina, estado seguido por Goiás, Acre, Bahia e Espírito Santo. Na Bahia e no Rio Grande do Norte, as classificaçôes evoluíram de média para baixa vulnerabilidade social; em contrapartida, nas UFs de Paraíba e Rondônia, as classificaçóes foram de baixa para média e muito baixa para baixa, respectivamente. 
Comparando os dois grupos e destacando a desigualdade existente entre eles, Roraima é a única UF em que o IVS para a população branca supera o IVS da população negra. Nessa UF, o IVS aumentou, entre 2017 e 2018, para as duas populaçóes.

O Amazonas segue em disparado como a UF mais desigual do país, tendo evidenciado ainda mais essa discrepância, seguido pelo Rio Grande do Sul, embora este tenha reduzido levemente a diferença entre os grupos no que se refere ao IVS.

Seguindo as análises com dados desagregados, a tabela 5 traz os dados do IVS para a população urbana. É possível observar que o Nordeste apresentou melhora do índice na maioria das UFs, sobretudo em Pernambuco e Rio Grande do Norte, tendo piorado apenas na Paraíba e em Sergipe, que, como vimos anteriormente, foram os dois estados que apresentaram aumento do IVS. Apesar dos avanços registrados na maioria de seus estados, a região ainda concentra os maiores IVS para a categoria urbana no país.

TABELA 5

Brasil: evolução do IVS da população urbana para as UFs e para o país (2017-2018)

\begin{tabular}{|c|c|c|}
\hline \multirow{2}{*}{ Região } & \multicolumn{2}{|c|}{ IVS } \\
\hline & 2017 & 2018 \\
\hline Brasil & 0,232 & 0,228 \\
\hline Distrito Federal & 0,255 & 0,249 \\
\hline Goiás & 0,244 & 0,233 \\
\hline Mato Grosso & 0,216 & 0,211 \\
\hline Mato Grosso do Sul & 0,195 & 0,193 \\
\hline Alagoas & 0,306 & 0,294 \\
\hline Bahia & 0,265 & 0,252 \\
\hline Ceará & 0,258 & 0,251 \\
\hline Maranhão & 0,305 & 0,304 \\
\hline Paraíba & 0,267 & 0,277 \\
\hline Pernambuco & 0,318 & 0,303 \\
\hline Piauí & 0,234 & 0,231 \\
\hline Rio Grande do Norte & 0,256 & 0,239 \\
\hline Sergipe & 0,255 & 0,268 \\
\hline Acre & 0,315 & 0,303 \\
\hline Amapá & 0,244 & 0,233 \\
\hline Amazonas & 0,324 & 0,328 \\
\hline Pará & 0,248 & 0,251 \\
\hline Rondônia & 0,171 & 0,182 \\
\hline Roraima & 0,201 & 0,213 \\
\hline Tocantins & 0,223 & 0,234 \\
\hline Espírito Santo & 0,232 & 0,220 \\
\hline Minas Gerais & 0,192 & 0,190 \\
\hline Rio de Janeiro & 0,282 & 0,276 \\
\hline São Paulo & 0,244 & 0,241 \\
\hline Paraná & 0,178 & 0,176 \\
\hline Rio Grande do Sul & 0,199 & 0,202 \\
\hline Santa Catarina & 0,122 & 0,116 \\
\hline
\end{tabular}

Fonte: Ipea, 2021. 
Na região Norte, apesar de os índices serem melhores que os observados para o Nordeste, houve aumento no IVS em cinco UFs, com melhora apenas para o Acre e o Amapá.

Com exceção do Amazonas (primeiro), que sofreu com o aumento de seu IVS na categoria, e Maranhão (segundo), com uma redução tímida, as demais UFs que compunham o ranking de maiores índices - Pernambuco (terceiro), Acre (terceiro) e Alagoas (quinto) apresentaram evoluçôes em seus índices, tendo a UF de Alagoas ascendido a sua classificação da faixa da média para a faixa da baixa vulnerabilidade social no meio urbano.

Santa Catarina segue apresentando o menor IVS urbano do país, tendo melhorado o índice entre 2017 e 2018. Além de Santa Catarina, Mato Grosso do Sul, Rondônia, Minas Gerais e Paraná também se situam na faixa da muito baixa vulnerabilidade social para suas populaçôes urbanas, configurando as melhores situaçóes observadas no país. 
